\title{
Valores genéticos de linhas puras de soja preditos com o uso do método de Papadakis
}

\author{
Lindolfo Storck ("); Nerinéia Dalfollo Ribeiro \\ Universidade Federal de Santa Maria, Departamento de Fitotecnia, Centro de Ciências Rurais, Av. Roraima, 1000, 97105-900 Santa \\ Maria (RS), Brasil. \\ (*) Autor correspondente: lindolfo@pq.cnpq.br
}

Recebido: 11/jan./2011; Aceito: 24/mar./2011

\begin{abstract}
Resumo
O método de Papadakis na estimação dos valores genéticos, em programas de melhoramento de soja, ainda é pouco usado. O objetivo deste trabalho foi avaliar as alterações do erro experimental e das estimativas dos valores genéticos causadas pelo método de Papadakis na análise estatística de experimentos de avaliação de linhas puras de soja. Foram usados os dados de produtividade de grãos de soja referentes a 216 ensaios de avaliação de linhas puras em diferentes estágios de melhoramento e de diferentes ciclos. Foi adotado o delineamento em blocos completos ao acaso e as unidades experimentais foram compostas por quatro fileiras de $6 \mathrm{~m}$ de comprimento, espaçadas a 0,4 m. Em cada ensaio foram obtidas as estimativas da média geral, das variâncias ambiental, genética e fenotípica média, dos coeficientes de variação, dos erros experimental e genético, do coeficiente de herdabilidade e da acurácia seletiva. As mesmas estimativas foram obtidas com o uso do método de Papadakis. As estimativas do coeficiente de variação genético, do coeficiente de herdabilidade média e da acurácia seletiva são mais elevadas com o uso do método de Papadakis, tanto em ensaios de cultivares de soja de ciclo precoce, médio e tardio, como nas misturas de ciclos. O método de Papadakis é recomendado nos programas de melhoramento de soja, pois permite estimativa de maior ganho na seleção entre linhagens avançadas.
\end{abstract}

Palavras-chave: Glycine max L., covariância, melhoramento de soja, erro experimental, precisão experimental.

\section{Soybean pure lines genetic values predicted by using the Papadakis method}

\begin{abstract}
The use of the Papadakis method to estimate the genetic values in soybean breeding programs is not frequent. The objective of this work was to quantify the alterations in the experimental errors and in estimation of the genetic values caused by the use of the Papadakis method, when analyzing trials with soybean pure lines. Yield data of pure lines related to 216 experiments, at different plant breeding stages and plant cycles (late, medium and early lines) were used. The experimental design was in randomized block and the experimental units were formed by four rows, six meters long and $0.4 \mathrm{~m}$ between rows. The general mean, environmental, genetic and phenotypic variances, genetic and experimental error variation coefficients, heritability coefficient and selective accuracy were obtained for each trial. The same variables were obtained by using the Papadakis method. The genetic values such as genetic variation coefficient, mean heritability coefficient and selective accuracy presented higher values with Papadakis method, regardless of plant breeding stages and maturity groups. The use of the method of Papadakis is recommended in soybean breeding programs improvement, which may improve the estimated genetic gain in selection between advanced pure lines.
\end{abstract}

Key words: Glycine max L., covariance, soybean improvement, experimental error, experimental precision.

\section{INTRODUÇÃO}

Novas cultivares de soja (Glycine max L.) proporcionaram ganho genético médio de $19 \mathrm{~kg} \mathrm{ha}^{-1} \mathrm{ano}^{-1}$, em um período de 40 anos de avaliaçấo (Rubin, 1996). Para o período de 20 anos, a produtividade de grãos de experimentos de avaliação de linhagens derivadas de quatro programas de melhoramento de soja no Rio Grande do Sul indica ganhos variando de zero a $71,5 \mathrm{~kg} \mathrm{ha}^{-1}$ ano $^{-1}$, dependendo do grupo de maturação e da região (Lange e Federizzi, 2009). Por outro lado, o nível de diversidade genética do germoplasma de soja utilizado em programas de melhoramento no Brasil, manteve-se constante nos últimos anos (Priolli et al., 2004). Como consequência, incrementos menores têm sido registrados e diferenças cada vez menores entre as linhagens devem ser detectadas para que os programas de melhoramento obtenham progresso com a seleção.

A avaliação das linhagens avançadas é uma etapa que requer grande demanda de trabalho, porque os experimentos precisam ser desenvolvidos em diferentes ambientes. O coeficiente de variação deve ser menor do 
que $20 \%$, exigência para a inscriçáo das novas cultivares de soja no Registro Nacional de Cultivares, no Ministério da Agricultura, Pecuária e Abastecimento (RNC-MAPA) (Brasil, 1998). Existem estudos das classes de coeficiente de variação experimental (Lúcio et al., 1999), diversas medidas da precisão experimental (CARGnelutti Filho et al., 2009) e técnicas para aumentar a precisão (MARTIN et al., 2005; 2007) para ensaios de produtividade de soja.

O método de Papadakis (PAPAdAkis, 1937) tem sido utilizado para diminuir o quadrado médio do erro e o coeficiente de variação em experimentos com feijão (SouzA et al., 2000), milho (CArgnelutti Filho et al., 2003) e soja (STORck et al., 2008 e 2009). Como esse método é embasado na análise de covariância e utiliza as médias móveis calculadas entre as parcelas vizinhas, este possibilita melhor discriminação entre os genótipos em ensaios de campo (CArgnelutti Filho et al., 2003; Storck et al., 2008). Além disso, o método de Papadakis se mostrou promissor na redução do erro experimental, com base na correlação entre parcelas adjacentes, quando se detectou heterogeneidade dentro dos blocos (Souza et al., 2000). Também, tem sido aplicado para melhorar as estimativas dos componentes da variância e da herdabilidade em linhagens avançadas de feijão (Costa et al., 2005). Estudos de simulação (PeArce, 1998) mostraram que o uso do método de Papadakis implica em estimativas dos contrastes não tendenciosas e uma importante redução na estimativa do erro experimental, principalmente em ensaios com formato mais retangular.

Ao analisar a expressão para a estimativa do coeficiente de herdabilidade $\left(\mathrm{h}^{2}\right)$, usado em nível de média de genótipo, constata-se a importância na redução da estimativa do erro experimental para aumentar a estimativa da $\mathrm{h}^{2}$. Sendo $\mathrm{h}^{2}$ relacionada com a medida de qualidade experimental denominada de acurácia seletiva, $A S=\sqrt{1-1 / F}=h^{2}$, em que $\mathrm{F}$ é o valor da estatística do teste $\mathrm{F}$ para genótipo, percebe-se que ambas dependem da magnitude da variação ambiental, do número de repetições e da proporção entre as variaçóes de natureza genética e ambiental (RESENDE e DUARTE, 2007) e tem a propriedade de informar a eficácia da inferência acerca do valor genético da cultivar, isto é, do seu valor de cultivo e uso (VCU) (REsEnde, 2002).

O emprego do método de Papadakis na estimação dos valores genéticos, em programas de melhoramento de soja, ainda é muito escasso. Assim, o objetivo deste trabalho foi avaliar as alterações do erro experimental e das estimativas dos valores genéticos causados pelo uso do método de Papadakis, na análise estatística de experimentos de avaliação de linhas puras de soja.

\section{MATERIAL E MÉTODOS}

Os dados de produtividade de grãos de soja, em $\mathrm{t} \mathrm{ha} \mathrm{h}^{-1} \mathrm{e}$ ajustados a 13\% de umidade, de 216 ensaios de avaliação de linhagens avançadas (Preliminar e valor de cultivo e uso) e de cultivares registradas (Recomendadas) foram utilizados. Todos os ensaios foram realizados em delineamento de blocos completos ao acaso, com número de repetições variando entre três e quatro e, de genótipos variando entre 10 e 20 . As unidades experimentais foram compostas por quatro fileiras de seis metros de comprimento, espaçadas a 0,4 $\mathrm{m}$, e área útil de 4,0 $\mathrm{m}^{2}$.

Os ensaios foram classificados quanto ao estágio de melhoramento (Preliminar, Valor de Cultivo e Uso e Recomendadas) e quanto ao ciclo (precoce, médio e tardio). O ensaio Preliminar foi constituído por genótipos de ciclos ainda não determinados. Os ensaios foram executados nos anos agrícolas de 2003/2004, 2004/2005 e 2005/2006, no conjunto de áreas experimentais (sete locais), coordenados pela Fundação Centro de Experimentação e Pesquisa (FUNDACEP - em Cruz Alta, Cachoeira do Sul e Restinga Seca) e pela Fundação Estadual de Pesquisa Agropecuária (FEPAGRO - em Júlio de Castilhos, Santo Augusto, São Borja e Veranópolis), no Estado do Rio Grande do Sul.

Em cada experimento foi procedida a análise de variância, considerando os blocos e os genótipos de efeitos aleatórios. Foram aplicados os testes de hipóteses para blocos e genótipos. Também, foi obtida a estimativa do quadrado médio de genótipos (QMg), do quadrado médio do erro (QMe), do número de repetiçóes $(\mathrm{J})$, das estimativas da média geral $(\hat{\mathrm{m}})$, da variância ambiental média $\left(\hat{\sigma}_{\mathrm{am}}^{2}=\mathrm{QMe} / \mathrm{J}\right)$, da variância genética média $\left(\hat{\sigma}_{\mathrm{gm}}^{2}=(\mathrm{QMg}-\mathrm{QMe}) / \mathrm{J}\right)$, do coeficiente de variação do erro experimental $(\mathrm{CVa}=100 \sqrt{\mathrm{QMe}} / \hat{\mathrm{m}})$, do coeficiente de variação genético $\left(\mathrm{CVg}=100 \sqrt{\hat{\sigma}_{\mathrm{gm}}^{2}} / \hat{\mathrm{m}}\right)$, coeficiente de herdabilidade para a média de genótipo $\left(\mathrm{h}^{2}=(\mathrm{QMg}-\mathrm{QMe}) / \mathrm{QMg}\right)$ e a acurácia seletiva, $A S=\sqrt{1-1 / F}$, em que $F$ é o valor da estatística do teste $\mathrm{F}$ para genótipo, obtida na análise de variância (Resende e Duarte, 2007).

Para cada um dos ensaios, considerando-se o delineamento como inteiramente casualizado, foi estimado o valor do erro experimental referente à parcela com o genótipo i na repetição j $\quad\left(\right.$ Erro $_{\mathrm{ij}}=$ Valor observado na parcela referente ao genótipo i da repetição $\mathrm{j}+$ média geral - média do genótipo i). Com esses valores, calculou-se o erro médio referente a cada parcela experimental como a média entre o erro da parcela considerada e os erros das parcelas experimentais vizinhas (lado direito, lado esquerdo, da frente e do fundo) existentes (CARgnelutti Filho et al., 2003). O valor do erro médio, de cada parcela experimental, é denominado de covariável para fins da análise da covariância (STEEL et al., 1997), segundo o delineamento inteiramente casualizado, o que corresponde à aplicação do método de PAPADAKIS (1937). Da análise da covariância, foram obtidas novamente as estimativas: $\hat{\mathrm{m}}, \hat{\mathrm{\sigma}}_{\mathrm{am}}^{2}, \hat{\mathrm{\sigma}}_{\mathrm{gm}}^{2}, C V a, C V g, \mathrm{~h}^{2}$ e AS, referentes a todos os experimentos.

Considerando que as estatísticas mais importantes para o melhoramento ( $\mathrm{CVa}, \mathrm{CVg}, \mathrm{h}^{2}$ e $\mathrm{AS}$ ), em geral, não seguem uma distribuição de probabilidade conhecida, 
adotou-se o método da reamostragem para a comparação entre os métodos de análise (com e sem o uso de Papadakis) e entre os ciclos ou entre os estágios de melhoramento. Assim, os valores das classes de ciclo e estágio foram comparados, dois a dois, pelo teste $\mathrm{t}$ - método de reamostragem bootstrap (MANLY, 1997), com 5000 simulaçóes para cada comparação, a 5\% de probabilidade de erro. Para isso, foi utilizado o aplicativo computacional BioEstat 5.0 (Ayres et al., 2007), em que são informados, por exemplo, os $\mathrm{N}$ valores de AS obtidos sem o uso do método de Papadakis (C1) e os N valores de AS obtidos com o uso do método de Papadakis (C2). Reamostras com reposição, de tamanho $\mathrm{N}$ em $\mathrm{C} 1$ e $\mathrm{C} 2$, para as estimativas das médias, das variâncias e a respectiva estatística t bootstrap são repetidas 5000 vezes para o respectivo teste de hipótese.

\section{RESULTADOS E DISCUSSÃO}

A variância entre blocos foi significativa $(\mathrm{p} \leq 0,05)$ em 63 ensaios $(29,16 \%)$, indicando que os blocos foram heterogêneos e o uso do delineamento de blocos ao acaso foi adequado. Por outro lado, em 153 ensaios (70,83\%) os blocos não foram heterogêneos. Nesse caso, os blocos não foram adequadamente posicionados ou a área experimental era homogênea. Embora na maioria dos 216 ensaios, o uso do delineamento inteiramente casualizado seja mais adequado, o uso de blocos deve continuar sendo preferido em experimentos de avaliação de linhagens avançadas e de cultivares registradas de soja, como uma estratégia para garantir o controle dessa fonte de erro experimental, em casos de necessidade técnica, como a conclusão da instalaçáo de um ou dois blocos do ensaio em dias diferentes. Além disso, a significância da variância entre blocos não interferiu na eficiência da aplicação do método de Papadakis (Tabela 1), pois as estimativas de AS são muito semelhantes.

Em relação aos 216 ensaios, o coeficiente de variação do erro experimental (CVa) variou de 3,5\% a 33,9\% no método de análise usual (sem Papadakis). Como a precisão experimental pode ser classificada como alta $(5,0 \%<\mathrm{CVa} \leq 9,0 \%)$ ou baixa $(15 \%<\mathrm{CVa} \leq 19 \%)$, de acordo com os limites estabelecidos por Lúcio et al. (1999), a faixa de variação da

Tabela 1. Valores (mínimo, máximo e média, $\mathrm{t} \mathrm{ha}^{-1}$ ) das estatísticas média geral $(\hat{\mathrm{m}})$, variância ambiental ( $\left.\hat{\mathrm{O}}_{\mathrm{am}}^{2}\right)$ e genética ( $\left.\hat{\mathrm{O}}_{\mathrm{gm}}^{2}\right)$ média, coeficiente de variação do erro experimental (CVa, \%) e genética (CVg, \%), coeficiente de herdabilidade média $\left(\mathrm{h}^{2}\right)$ e acurácia seletiva (AS) obtidas na análise de 216 ensaios de avaliação da produtividade de grãos de linhagens de soja, classificados em função do uso do método de Papadakis e da variabilidade de blocos

\begin{tabular}{|c|c|c|c|c|c|c|}
\hline \multirow{3}{*}{ Estatísticas } & \multicolumn{3}{|c|}{ Sem Papadakis } & \multicolumn{3}{|c|}{ Com Papadakis } \\
\hline & Mínimo & Máximo & Média & Mínimo & Máximo & Média \\
\hline & \multicolumn{6}{|c|}{ Geral (216 ensaios) } \\
\hline$\hat{\mathrm{m}}$ & 0,442 & 3,489 & 1,935 & 0,442 & 3,489 & 1,935 \\
\hline$\hat{\sigma}_{\mathrm{am}}^{2}$ & 0,00218 & 0,08515 & 0,02165 & 0,00084 & 0,05520 & 0,01213 \\
\hline$\hat{\sigma}_{\mathrm{gm}}^{2}$ & 0,00134 & 0,24616 & 0,04142 & 0,00319 & 0,24800 & 0,05060 \\
\hline $\mathrm{CVa}$ & 3,5 & 33,9 & 13,3 & 2,4 & 22,6 & 9,8 \\
\hline CVg & 2,7 & 30,1 & 10,5 & 3,5 & 32,0 & 11,7 \\
\hline$h^{2}$ & 0,13 & 0,93 & 0,62 & 0,34 & 0,97 & 0,78 \\
\hline \multirow[t]{2}{*}{ AS } & 0,36 & 0,97 & 0,77 & 0,59 & 0,98 & 0,88 \\
\hline & \multicolumn{6}{|c|}{ Blocos homogêneos (153 ensaios) } \\
\hline$\hat{\mathrm{m}}$ & 0,442 & 3,489 & 1,959 & 0,442 & 3,489 & 1,959 \\
\hline$\hat{\sigma}_{\mathrm{am}}^{2}$. & 0,00218 & 0,08053 & 0,02279 & 0,00084 & 0,04970 & 0,01249 \\
\hline$\hat{\sigma}_{\mathrm{gm}}^{2}$ & 0,00254 & 0,18378 & 0,04071 & 0,00566 & 0,21683 & 0,05158 \\
\hline CVa & 6,1 & 33,9 & 13,5 & 3,7 & 22,6 & 9,9 \\
\hline CVg & 2,7 & 30,1 & 10,4 & 4,3 & 32,0 & 11,8 \\
\hline$h^{2}$ & 0,13 & 0,92 & 0,61 & 0,46 & 0,96 & 0,79 \\
\hline \multirow[t]{2}{*}{ AS } & 0,36 & 0,96 & 0,77 & 0,68 & 0,98 & 0,88 \\
\hline & \multicolumn{6}{|c|}{ Blocos heterogêneos (63 ensaios) } \\
\hline$\hat{\mathrm{m}}$ & 0,609 & 3,065 & 1,876 & 0,609 & 3,065 & 1,876 \\
\hline$\hat{\sigma}_{\mathrm{am}}^{2}$. & 0,00332 & 0,08515 & 0,01887 & 0,00166 & 0,05521 & 0,01126 \\
\hline$\hat{\sigma}_{\mathrm{gm}}^{2}$ & 0,00134 & 0,24616 & 0,04314 & 0,00319 & 0,24800 & 0,04821 \\
\hline CVa & 3,5 & 24,1 & 12,8 & 2,4 & 18,1 & 9,7 \\
\hline CVg & 2,8 & 25,1 & 10,8 & 3,5 & 23,9 & 11,6 \\
\hline$h^{2}$ & 0,17 & 0,93 & 0,64 & 0,34 & 0,97 & 0,77 \\
\hline AS & 0,42 & 0,97 & 0,79 & 0,59 & 0,98 & 0,88 \\
\hline
\end{tabular}


precisão é ampla. Entretanto, o uso do método de Papadakis possibilitou a reduçáo da estimativa do CVa, e valores médios de $2,4 \%$ a $22,6 \%$ foram constatados. Portanto, um maior número de ensaios, todos aqueles com estimativa do coeficiente de variação inferior ou igual a $20 \%$, poderá ser aproveitado para fins de registro de cultivares de soja no RNC-MAPA (Brasil, 1998). Além disso, o limite máximo de estimativa do coeficiente de variação aceitável em relação à produtividade de grãos de soja é de 16\% (CARVALHo et al., 2003), devendo-se tomar os devidos cuidados com a interpretação da magnitude da estimativa do $\mathrm{CVa}$, conforme estudos sobre as medidas de precisão experimental de ensaios de feijão e soja (CARGnelutti Filho et al., 2009). Em experimentos com feijão (SouzA et al., 2000) e com milho (Cargnelutti Filho et al., 2003), a aplicação do método de Papadakis, também, foi promissora na redução do erro experimental.

A média da estimativa da variância genética média foi maior e a média da estimativa da variância ambiental média, menor, com o uso do método de Papadakis (Tabela 1) em todos os casos (geral, blocos homogêneos e blocos heterogêneos), o que se refletiu em estimativas de herdabilidade superiores. Estimativas de coeficientes de herdabilidade elevados estão associadas com maior variabilidade genética, maior acurácia seletiva (CARGNELUTTI FilHo et al., 2009) e maiores possibilidades de êxito na seleção de linhagens de soja com produtividade de grãos superior. Ademais, as estimativas dos contrastes entre médias de genótipos não são tendenciosas e têm menor variância (PeArCE, 1998). A utilização do método de Papadakis na avaliação de linhagens avançadas de feijão também resultou na alteração das estimativas dos componentes da variância e de herdabilidade (CosTA et al., 2005). Elevadas estimativas dos coeficientes de herdabilidade média, nos experimentos de avaliação, são garantia de seleção dos genótipos superiores daquele grupo de genótipos em avaliação. No entanto, não é garantia de que os genótipos selecionados sejam suficientemente superiores às cultivares usadas pelos produtores; por esse motivo, o ganho em produtividade pela substituição de cultivares produzidas pelas novas é pequena (Rubin, 1996; Priolli et al., 2004).

$\mathrm{O}$ uso do método de Papadakis reduziu significativamente $(\mathrm{p}<0,05)$ o CVa, nos dois estágios de melhoramento (Preliminar e VCU + Recomendadas) e não houve diferença entre os estágios (Tabela 2). Por outro lado, o $\mathrm{CVg}$ não modificou com o uso do método de Papadakis, mas estimativas maiores para o CVg no Ensaio Preliminar foram verificadas devido à maior estimativa da variância entre os genótipos nesta etapa do melhoramento e também às diferenças entre ciclos. No Ensaio Preliminar, o valor médio da estimativa de $h^{2}$ passou de $h^{2}=0,65$ para $^{2}=0,80$, com o uso do método de Papadakis (Tabela 2), representando um aumento de $15 \%$. Assim, para um ensaio de avaliação da produtividade, a expectativa de ganho na seleção, usando médias de linhagens corrigidas pelo método de Papadakis, fica acrescida em $15 \%$, isto é, terá $15 \%$ de ganho a mais em relação ao método usual. A média da estimativa da $\mathrm{h}^{2}$ deste estudo é próximo ao valor analisado por Үокоміzо e Vello (2000), de $\mathrm{h}^{2}=0,57$ para produtividade de grãos.

O Ensaio Preliminar é constituído por genótipos de diferentes ciclos (mistura de ciclos), pois nesse estágio os ciclos ainda não foram determinados e, por isto, a interação entre blocos e genótipos pode ser significativa, inflacionando as estimativas do erro experimental. Por esse motivo, a utilização de técnicas experimentais que permitam a redução do erro experimental e que possibilitam maior eficiência na quantificação dos valores genotípicos, deve ser avaliada para a cultura da soja. $\mathrm{O}$ método de Papadakis possibilitou maior precisão experimental (menor estimativa da variância ambiental, menor estimativa do coeficiente de variação do erro experimental e maior estimativa da acurácia seletiva) nos Ensaios Preliminares (Tabela 2). Assim, estimativas mais elevadas de variância genética, de coeficiente de variação genético e de coeficiente de herdabilidade média foram obtidos, possibilitando maior eficácia na seleção dos genótipos. Estimativas em níveis similares foram verificadas nos ensaios de VCU e de Cultivares Recomendadas, que foram desenvolvidos por grupo de maturação (precoce, médio e tardio) e, portanto, com maior uniformidade genética.

No conjunto dos 216 ensaios, as condiçóes de temperatura, de umidade, de fotoperíodo e seus efeitos sobre o crescimento e o desenvolvimento das plantas podem ser fatores determinantes para a definição do ciclo (precoce, médio e tardio) das cultivares de soja, conforme estudos de BARNI et al. (1985). As estimativas do coeficiente de variação genético, do coeficiente de herdabilidade média e da acurácia seletiva foram mais elevadas com o uso do método de Papadakis tanto em ensaios de cultivares de soja de ciclo precoce, médio, tardio como em mistura de ciclos (precoce, médio e tardio) (Tabela 3). Portanto, o método de Papadakis permitiu avaliar a variabilidade genética, independentemente do ciclo de maturação das cultivares de soja. Ao contrário, LANGE e Federizzi (2009), usando o método de análise usual, não detectaram avanço genético para o ciclo de maturação precoce em nenhuma das quatro regióes do Rio Grande do Sul. Entretanto, conforme Toledo et al. (2000), a baixa herdabilidade do caráter e os consideráveis níveis de interação entre genótipo e ambiente exigem que a avaliação da produtividade seja criteriosamente realizada para haver progresso genético por seleção. É justamente na avaliação criteriosa da produtividade das linhagens de soja que se justifica o uso do método de Papadakis. Podendo-se com este método, dimensionar os delineamentos experimentais em relação ao tamanho da parcela e número de repetiçôes (MARTin et al., 2005; 2007).

Pela visão geral dos resultados, observa-se que estimativas elevadas de variância genética, de coeficiente de variação genético, de coeficiente de herdabilidade e de acurácia seletiva e estimativas baixas de variância ambiental e de coeficiente de variação do erro experimental foram obtidos com o uso do método de Papadakis, no geral (Tabela 1), nos diferentes estágios de melhoramento (Tabela 2) e nos diferentes ciclos de 
Tabela 2. Número de ensaios ( $\mathrm{n})$ e médias das estatísticas: média geral $\left(\hat{\mathrm{m}}, \mathrm{t} \mathrm{ha}^{-1}\right)$, variância ambiental média $\left(\hat{\mathrm{O}}_{\mathrm{am}}^{2}\right)$ e genética média $\left(\hat{\mathrm{\sigma}}_{\mathrm{gm}}^{2}\right)$, coeficiente de variação do erro experimental $(\mathrm{CVa}, \%)$ e genética $(\mathrm{CVg}, \%)$, coeficiente de herdabilidade média $\left(\mathrm{h}^{2}\right)$ e acurácia seletiva (AS) obtidas na análise de 216 ensaios de avaliação da produtividade de grãos de linhagens de soja, classificado em funçáo do estágio do melhoramento (Preliminar, Valor de Cultivo e Uso - VCU - e Recomendadas) e do método de Papadakis

\begin{tabular}{|c|c|c|c|c|c|c|c|c|}
\hline \multirow{2}{*}{ Estágio } & n & $\hat{\mathbf{m}}$ & $\hat{\sigma}_{\mathrm{am}}^{2}$ & $\hat{\sigma}_{\mathrm{gm}}^{2}$ & $\mathrm{CVa}$ & $\mathrm{CVg}$ & $h^{2}$ & AS \\
\hline & \multicolumn{8}{|c|}{ Sem o uso do método de Papadakis } \\
\hline Preliminar & 129 & $1,828 b$ & 0,02157 & 0,04249 & $13,8 \mathrm{Aa}^{*}$ & $11,5 \mathrm{Aa}$ & $0,65 \mathrm{Ba}$ & $0,79 \mathrm{Ba}$ \\
\hline \multirow[t]{2}{*}{$V C U+\operatorname{Rec}$} & 87 & $2,095 \mathrm{a}$ & 0,02177 & 0,03983 & $12,6 \mathrm{Aa}$ & $9,0 \mathrm{Ab}$ & $0,57 \mathrm{Bb}$ & $0,74 \mathrm{Bb}$ \\
\hline & \multicolumn{8}{|c|}{ Com o uso do método de Papadakis } \\
\hline Preliminar & 129 & $1,828 \mathrm{~b}$ & 0,01201 & 0,05165 & $10,1 \mathrm{Ba}$ & $12,7 \mathrm{Aa}$ & $0,80 \mathrm{Aa}$ & $0,89 \mathrm{Aa}$ \\
\hline$V C U+\operatorname{Rec}$ & 87 & $2,095 \mathrm{a}$ & 0,01232 & 0,04905 & $9,5 \mathrm{Ba}$ & $10,3 \mathrm{Ab}$ & $0,76 \mathrm{Ab}$ & $0,87 \mathrm{Ab}$ \\
\hline
\end{tabular}

* Médias seguidas por letras diferentes (maiúscula entre método; minúscula entre estágio) diferem pelo teste $\mathrm{t}$ - método de reamostragem Bootstrap ( $\leq 5,05$ ).

Tabela 3. Número de ensaios $(\mathrm{n})$ e médias das estatísticas: média geral $\left(\hat{\mathrm{m}}, \mathrm{t}\right.$ ha $\left.{ }^{-1}\right)$, variância ambiental média $\left(\hat{\mathrm{\sigma}}_{\mathrm{am}}^{2}\right)$ e genética média $\left(\hat{\mathrm{\sigma}}_{\mathrm{gm}}^{2}\right)$, coeficiente de variação do erro experimental (CVa, \%) e genética $(\mathrm{CVg}, \%)$, coeficiente de herdabilidade média ( $\left.\mathrm{h}^{2}\right)$ e acurácia seletiva (AS) obtidas na análise de 216 ensaios de avaliaçáo da produtividade de grãos de linhagens de soja, classificado em função do ciclo e do método de Papadakis

\begin{tabular}{|c|c|c|c|c|c|c|c|c|}
\hline \multirow{2}{*}{ Ciclo } & $\mathbf{n}$ & $\hat{\mathbf{m}}$ & $\hat{\sigma}_{a m}^{2}$ & $\hat{\sigma}_{\mathrm{gm}}^{2}$ & $\mathrm{CVa}$ & CVg & $h^{2}$ & AS \\
\hline & \multicolumn{8}{|c|}{ Sem o uso do método de Papadakis } \\
\hline Precoce $(\mathrm{P})$ & 24 & 1,942 & 0,02108 & 0,04433 & $13,0 a^{*}$ & $9,7 \mathrm{a}$ & $0,58 \mathrm{~b}$ & $0,74 b$ \\
\hline Médio (M) & 22 & 2,000 & 0,01944 & 0,03680 & $13,1 \mathrm{a}$ & 8,9 a & $0,56 b$ & $0,73 b$ \\
\hline Tardio (T) & 22 & 1,978 & 0,01862 & 0,04831 & $12,7 \mathrm{a}$ & $10,8 \mathrm{a}$ & $0,68 \mathrm{~b}$ & $0,82 b$ \\
\hline \multirow[t]{2}{*}{ (P, M e T) } & 148 & 1,918 & 0,02252 & 0,04061 & $13,5 \mathrm{a}$ & $10,9 \mathrm{~b}$ & $0,62 b$ & $0,78 \mathrm{~b}$ \\
\hline & \multicolumn{8}{|c|}{ Com o uso do método de Papadakis } \\
\hline Precoce $(\mathrm{P})$ & 24 & 1,942 & 0,01207 & 0,04919 & $9,9 \mathrm{~b}$ & $10,7 \mathrm{a}$ & $0,75 \mathrm{a}$ & $0,86 a$ \\
\hline Médio (M) & 22 & 2,000 & 0,01008 & 0,04721 & 9,4 b & $10,4 \mathrm{a}$ & $0,77 \mathrm{a}$ & $0,88 a$ \\
\hline Tardio (T) & 22 & 1,978 & 0,01156 & 0,05536 & $10,0 \mathrm{~b}$ & $11,7 \mathrm{a}$ & $0,80 \mathrm{a}$ & $0,89 a$ \\
\hline$(\mathrm{P}, \mathrm{M}$ e $\mathrm{T})$ & 148 & 1,918 & 0,01254 & 0,05063 & $9,9 \mathrm{~b}$ & $12,1 \mathrm{a}$ & $0,79 a$ & $0,89 a$ \\
\hline
\end{tabular}

* Médias seguidas por letras diferentes, entre método/por ciclo, diferem pelo teste t - método de reamostragem Bootstrap (p $\leq 0,05)$.

maturação (Tabela 3) para os 216 ensaios de avaliação da produtividade de grãos. Por isso, sua utilização na análise de dados provenientes de programas de melhoramento de soja é recomendável, pois permitirá uma seleção não tendenciosa entre as linhagens avançadas, com alta precisão experimental.

\section{AGRADECIMENTOS}

Ao Conselho Nacional de Desenvolvimento Científico e Tecnológico (CNPq) pelas bolsas concedidas e às Instituições FUNDACEP (Cruz Alta) e FEPAGRO Sementes (Júlio de Castilhos) pela cessão dos dados.

\section{REFERÊNCIAS}

AYRES, M.; AYRES Jr., M.; AYRES, D.L.; SANTOS, A.A.S. BioEstat - Aplicaçốes estatísticas nas áreas das ciências biomédicas. 2007. URL. <http://rs579.rapidshare.com/files/171642982/ BioEstat.zip> Acesso em 23 fev. 2010.

BARNI, N.A.; GOMES, J.E.S.; HILGERT, E.R. Épocas de semeadura de cultivares de soja para o Rio Grande do Sul. IPAGRO Informa, n.28, p.25-30, 1985.
BRASIL - MINISTÉRIO DA AGRICULTURA E DO ABASTECIMENTO. ANEXO VI. Requisitos mínimos para determinação do valor de cultivo e uso de soja (Glycine max L.), para a inscrição no registro nacional de cultivares - RNC. 1998.

CARGNELUTTI FILHO, A.; STORCK, L.; LÚCIO, A.D. Ajustes de quadrado médio do erro em ensaios de competição de cultivares de milho pelo método de Papadakis. Pesquisa Agropecuária Brasileira, v.38, p.467-473, 2003.

CARGNELUTTI FILHO, A.; STORCK, L.; RIBEIRO, N.D. Medidas da precisão experimental em ensaios com genótipos de feijão e de soja. Pesquisa Agropecuária Brasileira, v.44, p.12251231, 2009.

CARVALHO C.G.P.; ARIAS, C.A.A.; ALMEIDA, J.L.A.; KIIHL, R.A.S.; OLIVEIRA, M.F.; HIROMOTO, D.M.; TAKEDA, C. Proposta de classificação dos coeficientes de variação em relação à produtividade e altura da planta de soja. Pesquisa Agropecuária Brasileira, v.38, p.187-193, 2003.

COSTA, J.R.; BUENO FILHO, J.S.; RAMALHO, M.A.P. Análise espacial e de vizinhança no melhoramento genético de plantas. Pesquisa Agropecuária Brasileira, v.40, p.1073-1079, 2005.

LANGE, C.E.; FEDERIZZI, L.C. Estimation of soybean genetic progress in the South of Brazil using multi-environmental yield trials. Scientia Agricola, v.66, p.309-316, 2009. 
LÚCIO, A.D.; STORCK, L.; BANZATTO, D.A. Classificação dos experimentos de competiçáo de cultivares quanto a sua precisão. Pesquisa Agropecuária Gaúcha, v.5, p.99-103, 1999.

MANLY, B.F.J. Randomization, bootstrap and Monte Carlo methods in biology. $2^{\text {nd }}$ ed. New Zealand: University of Otaga, 1997. 356p.

MARTIN, T.N.; DUTRA, L.M.C.; JAUER, A.; STORCK, L.; ZABOT, L.; UHRY, D.; SANTI, A.L.; STEFANELO, C.; LUCCA FILHO, O.A. Tamanho ótimo de parcela e número de repetições em soja (Glycine max). Ciência Rural, v.35, p.271276, 2005.

MARTIN, T.N.; JAUER, A.; DUTRA, L.M.C.; SANTI, A.L.; ZABOT, L. Metodologia experimental para rendimento de grãos de soja em condiçôes de restrição de espaço. Bragantia, v.66, p.521526, 2007.

PAPADAKIS, J.S. Méthod statistique pour des experiences sur champ. Thessalonike: Institut d'Amélioration des Plantes à Salonique, 1937.30 p. (Bullettin, 23)

PEARCE, S.C. Field experimentation on rough land: the method of Papadakis reconsidered. Journal of Agricultural Science, v.131, p.1-11, 1998.

PRIOLLI, R.H.G.; MENDES-JUNIOR, C.T.; SOUSA, S.M.B.; SOUSA, N.E.A.; CONTEL, E.P.B. Diversidade genética da soja entre períodos e entre programas de melhoramento no Brasil. Pesquisa Agropecuária Brasileira, v.39, p.967-975, 2004.

RESENDE, M.D.V. Genética biométrica e estatística no melhoramento de plantas perenes. Brasília: Embrapa Informação Tecnológica, 2002. 975p.
RESENDE, M.D.V.; DUARTE, J.B. Precisão e controle de qualidade em experimentos de avaliação de cultivares. Pesquisa Agropecuária Tropical, v.37, p.182-197, 2007.

RUBIN, S.A.L. Progresso do melhoramento genético da soja no Estado do Rio Grande do Sul. Pesquisa Agropecuária Gaúcha, v.2, p.139-147, 1996.

SOUZA, E.; GERALDI, I.O.; RAMALHO, M.A.P. Alternativas experimentais na avaliação de famílias em programas de melhoramento genético de feijoeiro. Pesquisa Agropecuária Brasileira, v.35, p.1765-1771, 2000.

STEEL, R.G.D.; TORRIE, J.H.; DICKEY, D.A. Principles and procedures of statistics: a biometrical approach. 3.ed. New York: McGraw Hill Book, 1997. 666p.

STORCK, L.; CARGNELUTTI FILHO, A.; LÚCIO, A.D.; LOPES, S.J. Método de Papadakis e número de repetiçôes em experimentos de soja. Ciência Rural, v.39, p.977-982, 2009.

STORCK, L.; STECLING, C.; ROVERSI, T.; LOPES, S.J. Utilizaçáo do método de Papadakis na melhoria da qualidade experimental de ensaios de soja. Pesquisa Agropecuária Brasileira, v.43, p.581-587, 2008.

TOLEDO, J.F.F.; ARIAS, C.A.A.; OLIVEIRA, M.F.; TRILLER, C.; MIRANDA, Z.F.S. Genetic and environmental analyses of yield in six biparental soybean crosses. Pesquisa Agropecuária Brasileira, v.35, p.1783-1796, 2000.

YOKOMIZO, G.K.; VELLO, N.A. Coeficiente de determinação genotípica e de diversidade genética em topocruzamentos de soja tipo alimento com tipo grão. Pesquisa Agropecuária Brasileira, v.35, p.2223-2228, 2000. 